\title{
LOWER 2-ESTIMATES FOR SEQUENCES IN BANACH LATTICES
}

\author{
FRANK RÄBIGER
}

(Communicated by William J. Davis)

\begin{abstract}
We characterize Banach lattices in which every bounded sequence contains a subsequence which either converges in norm or satisfies a lower 2 estimate. As a consequence we obtain, for the class of all Banach lattices, a positive answer to a question of D.J. Aldous and D.H. Fremlin whether a Banach space of cotype 2 satisfies the above-mentioned property.
\end{abstract}

D. J. Aldous and D. H. Fremlin proved that every $A L$-space $E$ has the following property (cf. [1, Theorem 6]):

Every bounded sequence $\left(x_{n}\right)$ in $E$ has a subsequence which either converges in norm or satisfies a lower 2-estimate.

In this note we characterize the Banach lattices satisfying $(A F)$. As a consequence we obtain that every Banach lattice of cotype 2 has property $(A F)$ which solves a problem of D. J. Aldous and D. H. Fremlin (cf. [1, §9]) for the class of all Banach lattices.

Our notation is standard and follows [3]. Let us recall the following definitions. A sequence $\left(x_{n}\right)$ in a Banach space $E$ is called semi-normalized if it is bounded and $\inf _{n}\left\|x_{n}\right\|>0$. A sequence $\left(x_{n}\right)$ in $E$ satisfies a lower 2-estimate if there exists $c>0$ such that for every $n \in \mathbf{N}$ and every choice of scalars $\alpha_{1}, \ldots, \alpha_{n} \in \mathbf{R}$, we have $c\left(\sum_{k=1}^{n}\left|\alpha_{k}\right|^{2}\right)^{1 / 2} \leq\left\|\sum_{k=1}^{n} \alpha_{k} x_{k}\right\|$. A sequence $\left(x_{n}\right)$ in a Banach lattice $E$ is called disjoint if $\inf \left(\left|x_{n}\right|,\left|x_{m}\right|\right)=0$ for all $n, m \in \mathbf{N}$ with $n \neq m$.

We begin with a lemma which occurs implicitly in the proof of Theorem 6 in [1].

Lemma. Let $E$ be a weakly sequentially complete Banach space. Suppose every semi-normalized weakly null sequence in $E$ contains a subsequence satisfying a lower 2-estimate. Then $E$ has property $(A F)$.

Proof. Let $\left(x_{n}\right)$ be a bounded sequence in $E$ such that no subsequence is norm convergent. If $\left(x_{n}\right)$ does not have a weak Cauchy subsequence, then, by Rosenthal's $l_{1}$-theorem (cf. $[2,2 . e .5]$ ), there is a subsequence equivalent to the

Received by the editors May 26, 1989.

1980 Mathematics Subject Classification (1985 Revision). Primary 46B20, 46B30.

Key words and phrases. Banach lattice, lower 2-estimate, cotype 2. 
unit vector basis of $l_{1}$, which obviously satisfies a lower 2-estimate. Otherwise there exists a weak Cauchy subsequence $\left(y_{n}\right)$ of $\left(x_{n}\right)$. Since $E$ is weakly sequentially complete, $\left(y_{n}\right)$ converges weakly to some $y \in E$ but $\left(y_{n}\right)$ does not converge in norm. Our assumption implies that a subsequence of $\left(y_{n}-y\right)$ satisfies a lower 2-estimate, hence, by Lemma 2 in [1], a subsequence of $\left(y_{n}\right)$ satisfies a lower 2-estimate.

We are already in the position to state our main result.

Theorem. Let $E$ be a Banach lattice. Then the following assertions are equivalent :

(a) $E$ satisfies $(A F)$.

(b) Every semi-normalized disjoint sequence in $E$ contains a subsequence which satisfies a lower 2-estimate.

Proof. (a) $\Rightarrow(\mathrm{b})$. Every disjoint norm convergent sequence in a Banach lattice converges to zero. Hence a semi-normalized disjoint sequence $\left(x_{n}\right)$ in $E$ cannot have a convergent subsequence. Since $E$ satisfies $(A F)$ there is a subsequence of $\left(x_{n}\right)$ which satisfies a lower 2-estimate and assertion (b) follows.

(b) $\Rightarrow(\mathrm{a})$. The assumption on $E$ implies that $E$ does not contain a Banach sublattice isomorphic to $c_{0}$. As a consequence $E$ is weakly sequentially complete [3, Remark on p. 35, 1.c.4]. By the lemma, it is enough to show that, for a semi-normalized weakly null sequence $\left(x_{n}\right)$ in $E$ there is a subsequence which satisfies a lower 2-estimate. We may assume that $E$ is separable, otherwise we consider the closed separable sublattice of $E$ generated by $\left(x_{n}\right)$. Since $E$ is weakly sequentially complete, the Banach lattice $E$ has order continuous norm $[3,1 . c .4,1 . a .8]$. There exists a probability space $(\Omega, \Sigma, \mu)$ such that $E$ can be identified as a vector lattice with an ideal of $L_{1}(\Omega, \Sigma, \mu)$, and with this identification we have $\|j x\|_{1} \leq\|x\|_{E}$ for all $x \in E$, where $j: E \rightarrow L_{1}(\Omega, \Sigma, \mu)$ denotes the canonical injection (cf. [3, 1.b.14]). Suppose there exists $\varepsilon>0$ such that $\varepsilon\left\|x_{n}\right\|_{E} \leq\left\|j x_{n}\right\|_{1}$ for every $n \in \mathbf{N}$. Then $\left(j x_{n}\right)$ is a semi-normalized weakly null sequence in $L_{1}(\Omega, \Sigma, \mu)$. Since $L_{1}(\Omega, \Sigma, \mu)$ has property $(A F)$ [1, Theorem 6], a subsequence of $\left(j x_{n}\right)$ and then also a subsequence of $\left(x_{n}\right)$ satisfies a lower 2-estimate. If such an $\varepsilon>0$ does not exist we can find a subsequence $\left(x_{n_{k}}\right)$ of $\left(x_{n}\right)$ and a disjoint sequence $\left(y_{k}\right)$ in $E$ such that $\left\|x_{n_{k}}-y_{k}\right\|_{E} \leq 2^{-k}, k \in \mathbf{N}$ (cf. [3, Proofs of $1 . c .10$ and 1.c.8]). Our assumption implies that there is a subsequence of $\left(y_{k}\right)$ satisfying a lower 2-estimate. Since $\Sigma_{k}\left\|x_{n_{k}}-y_{k}\right\|_{E}<\infty$ there exists a subsequence of $\left(x_{n_{k}}\right)$ which also satisfies a lower 2-estimate [1, Lemma 2]. This proves the theorem.

D. J. Aldous and D. H. Fremlin [1, §9] asked whether every Banach space of cotype 2 has property $(A F)$. From the Theorem we get that at least for Banach lattices this implication holds. We recall the following notions.

Let $E$ be a Banach lattice. Then $E$ has cotype $q$ for some $q \geq 2$ if there exists a constant $M>0$ such that, for every $n \in \mathbf{N}$ and arbitrary vectors 
$x_{1}, \ldots, x_{n} \in E$, we have

$$
M\left(\sum_{k=1}^{n}\left\|x_{k}\right\|^{q}\right)^{1 / q} \leq \int_{0}^{1}\left\|\sum_{k=1}^{n} r_{k}(t) x_{k}\right\| d t,
$$

where $\left(r_{k}\right)$ denotes the sequence of Rademacher functions on the interval $[0,1][3,1 . e .12]$. The Banach lattice $E$ satisfies a lower q-estimate, $1 \leq q<\infty$, if there exists a constant $c>0$ such that, for every $n \in \mathbf{N}$ and every choice of pairwise disjoint elements $x_{1}, \ldots, x_{n} \in E$, we have

$$
c\left(\sum_{k=1}^{n}\left\|x_{k}\right\|^{q}\right)^{1 / q} \leq\left\|\sum_{k=1}^{n} x_{k}\right\|
$$

[3, 1.f.4]. If $x_{1}, \ldots, x_{n}$ are pairwise disjoint elements of a Banach lattice $E$ then, for an arbitrary choice of signs $\varepsilon_{k} \in\{-1,1\}, 1 \leq k \leq n$, we have $\left\|\sum_{k=1}^{n} \varepsilon_{k} x_{k}\right\|=\left\|\sum_{k=1}^{n} x_{k}\right\|$. Since the Rademacher functions take values in $\{-1,1\}$ we obtain $\int_{0}^{1}\left\|\sum_{k=1}^{n} r_{k}(t) x_{k}\right\| d t=\left\|\sum_{k=1}^{n} x_{k}\right\|$. As a consequence every Banach lattice $E$ of cotype $q, q \geq 2$, satisfies a lower $q$-estimate. On the other hand every Banach lattice $E$ which satisfies a lower 2-estimate fulfills assertion (b) of the Theorem.

Hence, the Theorem implies the following result.

Corollary. Let $E$ be a Banach lattice of cotype 2 , then $E$ satisfies $(A F)$.

The cotype 2 condition is not necessary for a Banach lattice to have property $(A F)$. For instance, $E:=\oplus_{l_{1}} l_{p_{n}}^{n}$, the $l_{1}$-sum of the sequence of spaces $\left(\mathbf{R}^{n}\right.$, $\left.\|\cdot\|_{p_{n}}\right)$, satisfies $(A F)$ for every sequence $\left(p_{n}\right)$ in $[1, \infty]$ (cf. [4, Corollary 2.5, 1.3 Example (b)]). However, if $\varlimsup_{n} p_{n}=\infty$ then $E$ has cotype $\infty$.

\section{REFERENCES}

1. D. J. Aldous and D. H. Fremlin, Colacunary sequences in L-spaces, Studia Math. 71 (1982), 297-304.

2. J. Lindenstrauss and L. Tzafriri, Classical Banach spaces I. Sequence spaces, SpringerVerlag, Berlin, Heidelberg, and New York, 1977.

3. __ Classical Banach Spaces II. Function spaces, Springer-Verlag, Berlin, Heidelberg, and New York, 1979.

4. F. Räbiger, Lower and upper 2-estimates for order bounded sequences and Dunford-Pettis operators between certain classes of Banach lattices (Proc. Univ. of Texas at Austin 19871989), Lecture Notes in Math, Springer-Verlag (to appear).

Mathematisches Institut, Universität Tübingen, Auf Der Morgenstelle 10, D-7400 Tübingen, Federal Republic of Germany 